\title{
Comunicação
}

\section{Fungos micorrízicos arbusculares afetando a produção de mudas de pinhão-manso na região Sul do Estado de Tocantins, Brasil}

\author{
Rita de Cássia Cunha Saboya ${ }^{1}$, Aloisio Freitas Chagas Jr ${ }^{2 *}$, Fábio Pinto dos Reis Monteiro \\ Gil Rodrigues dos Santos ${ }^{2}$ Eduardo Andrea Lemus Erasmo ${ }^{2}$, Lillian França Borges Chagas ${ }^{4}$
}

\section{RESUMO}

O presente trabalho teve como objetivo avaliar os efeitos da inoculação de quatro espécies de fungos micorrízicos arbusculares no crescimento e nutrição de mudas de pinhão-manso. Foram utilizados 12 tratamentos e 10 repetições, sendo: a inoculação individual (Scutellospora calospora, Glomus clarum, Gigaspora margarita, Acaulospora morrowiae), em dupla das espécies (S. calospora + G. clarum, S. calospora + G. margarita, S. calospora + A. morrowiae, G. clarum + G. margarita, G. clarum + A. morrowiae e G. margarita + A. morrowiae), com a mistura das quatro espécies de micorrizas (MIX) e a testemunha sem inoculação com micorrizas. Para os teores de macro e micronutrientes, 75 dias após a emergência das plantas, diferenças significativas $(p<0,05)$ foram observadas para os teores de nitrogênio $(\mathrm{N})$, fósforo $(\mathrm{P})$, potássio $(\mathrm{K})$, magnésio $(\mathrm{Mg})$, enxofre $(\mathrm{S})$, manganês $(\mathrm{Mn})$ e boro $(\mathrm{B})$, com destaque para a inoculação com G. clarum e A. morrowiae isoladamente e em dupla inoculação. Para a biomassa da parte aérea e raiz, colonização micorrízica e eficiência micorrízica, as maiores médias ( $\mathrm{p}>0,005)$ foram observadas para os tratamentos com G. clarum e A. morrowiae inoculados isoladamente e para a dupla inoculação de $S$. calospora $+G$. clarum e G. clarum + A. morrowiae.

Palavras-chave: Jatropha curcas L., endomicorrizas, produção de mudas.

\section{ABSTRACT}

\section{Arbuscular mycorrhizal fungi inoculation on physic nut seedling production in Southern Tocantins State, Brazil}

This study aimed to evaluate the effects of the inoculation of four species of arbuscular mycorrhizal fungi on growth and nutrient status of seedlings of Jatropha curcas L. The experiment consisted of 12 treatments and 10 replicates inoculated with one and two of the mycorrhizal species Scutellospora calospora, Glomus clarum, Gigaspora margarita, Acaulospora morrowiae, with a mixture of the four species (MIX) and a non-inoculated control. After 75 days of plant emergence, significant differences $(\mathrm{P}<0.05)$ were observed for contents 'of nitrogen $(\mathrm{N})$, phosphorus $(\mathrm{P})$, potassium $(\mathrm{K})$, magnesium $(\mathrm{Mg})$, sulfur $(\mathrm{S})$, manganese $(\mathrm{Mn})$ and boron $(\mathrm{B})$, especially for the inoculation with $G$. clarum and A. morrowiae, both alone and dual inoculation. The highest means $(\mathrm{P}<0.05)$ of biomass of shoots and root, mycorrhizal colonization and mycorrhizal efficiency were found in the treatment with G. clarum and A. morrowiae inoculated separately and for the dual inoculation of S. calospora + G. clarum and G. clarum + A. morrowiae.

Key words: Jatropha curcas L., endomycorrhiza, seedling production.

\footnotetext{
Recebido para publicação em 05/11/2010 e aprovado em 13/12/2011

'Engenheira-Agrônoma. Embrapa Transferência de Tecnologia SNTENCG, Parque Estação Biológica PqEB s/nº, $70770-901$ Brasília, Distrito Federal, Brasil. cassia.saboya@cnpa.embrapa.br

${ }^{2}$ Engenheiros-Agrônomos, Doutores. Universidade Federal do Tocantins, Campus Universitário de Gurupi, Rua Badejós, Chácaras 69/72 Lt. 07, Zona Rural, Caixa Postal 66, 77404970 Gurupi, Tocantins, Brasil. chagasjraf@uft.edu.br; gilrsan@uft.edu.br; dirgurupi@mail.uft.edu.br (*Autor para correspondência.)

${ }^{3}$ Engenheiro-Agrônomo. Mestrando da Universidade Federal do Tocantins, Campus Universitário de Gurupi, Rua Badejós, Chácaras 69/72 Lt. 07, Zona Rural, Caixa Postal 66, 77404-970 Gurupi, Tocantins, Brasil. agrofprm@hotmail.com

${ }^{4}$ Engenheira-Agrônoma, Mestre. Universidade Federal do Tocantins, Campus Universitário de Gurupi, Rua Badejós, Chácaras 69/72 Lt. 07, Zona Rural, Caixa Postal 66, 77404970 Gurupi, Tocantins, Brasil. lillianfb@hotmail.com
} 


\section{INTRODUÇÃO}

O pinhão-manso (Jatropha curcas L.) é um arbusto de crescimento rápido, cujo ciclo produtivo pode chegar a 40 anos e atingir produtividade média de $2 \mathrm{t} \mathrm{ha}^{-1}$ de sementes (Dias et al., 2007). Seu percentual de óleo por semente gira em torno de $40 \%$, e de alta qualidade, possuindo muitas qualidades para produção de biodiesel (Francis et al., 2005). Porém, tem-se a necessidade de buscar alternativas adequadas para o desenvolvimento dessa cultura, possibilitando maiores produtividades e, consequentemente, maior retorno econômico.

Uma interessante tecnologia que possibilita o desenvolvimento das plantas, mesmo em solos pobres e degradados, e que permite diminuir a utilização de insumos, é a exploração da simbiose micorrízica por meio da inoculação das plantas com fungos micorrízicos arbusculares (FMAs) capazes de contribuir para o crescimento vegetal (Miranda, 2008; Folli-Pereira et al., 2010). Uma vez estabelecida a associação micorrizas-planta e desenvolvida (Kiriachek et al., 2009), os FMAs contribuem para o crescimento de culturas, sobretudo em solos com baixo teor de nutrientes, como os da região do Cerrado (Miranda, 2008).

Por se tratar de uma espécie arbórea, a inoculação do pinhão-manso com FMAs, já na fase de formação de mudas, pode assegurar sua boa formação, garantindo o estabelecimento posterior de FMA no campo e, consequentemente, os benefícios inerentes dessa associação (Carvalho, 2008). Assim, este trabalho teve como objetivo avaliar os efeitos da inoculação de quatro espécies de FMAs no crescimento e desenvolvimento inicial de mudas de pinhão-manso produzidas em viveiros em Gurupi, Sul do Estado do Tocantins.

\section{MATERIAL E MÉTODOS}

A pesquisa foi conduzida em casa de vegetação na Universidade Federal do Tocantins, Campus de Gurupi, Estado de Tocantins, localizado a $11^{\circ} 43^{\prime} 45^{\prime \prime}$ de latitude Sul e 49 04' 07' longitude Oeste. O solo utilizado no experimento foi um Latossolo Vermelho-Amarelo distrófico, misturado na proporção 1/1 (v/v) com esterco, cujas características químicas e físicas estão apresentadas na Tabela 1. O solo misturado ao esterco foi autoclavado por duas vezes em temperatura de $120^{\circ} \mathrm{C}$, por duas horas.
Foram utilizados 12 tratamentos, sendo: quatro correspondentes à inoculação individual de cada espécie de fungo micorrízico (Scutellospora calospora, Glomus clarum, Gigaspora margarita, Acaulospora morrowiae), seis em combinação de duas espécies diferentes de micorrizas (S. calospora + G. clarum, S. calospora $+G$. margarita, S. calospora + A. morrowiae, G. clarum $+G$. margarita, G. clarum + A. morrowiae e G. margarita $+A$. morrowiae), um com a mistura das quatro espécies de micorrizas (MIX) e uma testemunha sem inoculação com os fungos micorrízicos, em um delineamento inteiramente casualizado, e 10 repetições.

Foi utilizada uma planta por vaso com capacidade de 10 $\mathrm{kg}$ de solo. A inoculação foi realizada utilizando-se $2 \mathrm{~g}$ de solo-inóculo de cada FMA, por vaso, com cerca de 160 esporos, obtidos na Embrapa Agrobiologia. No tratamento com a inoculação de todas as espécies de FMA (MIX) foi empregado 0,5 g de solo-inóculo de cada FMA, totalizando $2 \mathrm{~g}$ de solo-inóculo. Para tentar uniformizar os tratamentos quanto aos demais componentes da microbiota do inóculo, adicionaram-se, em todos os recipientes, $10 \mathrm{~mL}$ de um filtrado isento de propágulo de FMA, preparado com os solosinóculos dos quatro FMAs testados. A inoculação com FMA foi realizada no momento do plantio.

Setenta e cinco dias após a emergência, as plantas foram colhidas inteiras para avaliação, quando foi determinada a produção de massa de matéria seca na parte aérea (MSPA), nas raízes (MSR) e total (MST), a partir da secagem em estufa a $65^{\circ} \mathrm{C}$ até peso constante. $\mathrm{Na}$ caracterização química da parte aérea foram analisados os teores de $\mathrm{N}$ total, pelo método Kjeldahl (Bremner \& Mulvaney, 1982). Os elementos totais de S, Fe, Mn, B, $\mathrm{Zn}$, e $\mathrm{Cu}$ foram extraídos por meio de digestão ácida, com os ácidos nítrico e perclórico (Lamothe et al., 1986), e determinados por espectrometria de emissão em plasma; $\mathrm{Ca}$ e $\mathrm{Mg}$ por espectrofotometria de absorção atômica; K por espectrometria de emissão em chama; e P total por colorimetria.

As análises da colonização radicular por FMA em lâminas foram estimadas em 100 fragmentos de $1 \mathrm{~cm}$, determinadas segundo Giovanetti \& Mosse (1980).

Os dados obtidos foram analisados pelo teste ScottKnott, a 0,05\% de probabilidade, utilizando-se o programa estatístico Sisvar.

Tabela 1. Análises química e física do substrato composto de solo Latossolo Vermelho-Amarelo distrófico misturado na proporção $1 / 1(\mathrm{v} / \mathrm{v})$ com esterco

\begin{tabular}{|c|c|c|c|c|c|c|c|c|c|}
\hline $\mathrm{Ca}+\mathrm{Mg}$ & $\mathbf{C a}$ & Mg & Al & $\mathrm{H}+\mathrm{Al}$ & $\mathbf{K}$ & CTC (t) & SB & $\mathbf{P}$ & $\mathbf{V}$ \\
\hline \multicolumn{8}{|c|}{$\mathrm{cmol} / \mathrm{dm}^{3}$} & $\mathrm{mg} / \mathrm{dm}^{3}$ & $\%$ \\
\hline 3,7 & 3,0 & 0,7 & 0,1 & 2,5 & 0,2 & 6,3 & 3,9 & 90 & 60,9 \\
\hline \multicolumn{2}{|c|}{ MO (\%) } & \multicolumn{2}{|c|}{$\mathrm{pH}\left(\mathrm{H}_{2} \mathrm{O}\right)$} & \multicolumn{2}{|c|}{ Areia $(\%)$} & \multicolumn{2}{|c|}{ Silte $(\%)$} & Argila (\%) & \\
\hline \multicolumn{2}{|c|}{5,0} & \multicolumn{2}{|c|}{5,8} & \multicolumn{2}{|c|}{69,0} & \multicolumn{2}{|c|}{8,8} & 22,2 & \\
\hline
\end{tabular}




\section{RESULTADOS E DISCUSSÃO}

Para os teores de macronutrientes na parte aérea (Tabela 2) não foram observadas diferenças significativas dos teores de cálcio $(\mathrm{Ca})$.

Quanto ao nitrogênio $(\mathrm{N})$, os melhores resultados foram encontrados para os tratamentos com $S$. calospora, G. clarum, A. morrowiae, G. margarita + A. morrowiae, ( $\mathrm{p}<0,05$ ), com incremento no teor de $\mathrm{N}$ de 17,4 a 45,4\% em relação ao tratamento testemunha sem FMA (Tabela 2). Para os teores de fósforo $(\mathrm{P})$, os maiores valores $(\mathrm{p}<0,05)$ foram observados nos tratamentos com G. clarum, A. morrowiae, G. clarum + G. margarita e G. clarum $+A$. morrowiae, com incremento no teor de $\mathrm{P}$ variando de $49 \mathrm{a}$ 63,5\% em relação à testemunha sem FMA (Tabela 2), demonstrando o efeito positivo da associação do pinhãomanso com FMA quanto à absorção do $\mathrm{N}$ e $\mathrm{P}$. Balota et al. (2010) também encontraram teores de $\mathrm{P}$ na parte aérea maiores em plantas micorrizadas, com acréscimos acima de $100 \%$ em relação à testemunha sem FMA.

Os teores de $\mathrm{P}$ encontrados no presente trabalho foram superiores aos constatados por Silva et al. (2010) em trabalho com pinhão-manso, em casa de vegetação, com adubação completa e omissão do nutriente, porém sem a utilização de FMA. Efeitos positivos da inoculação com FMA sobre o crescimento e utilização de P são comuns na literatura, sendo considerado o resultado da ação do micélio externo às raízes que aumentam a superfície de absorção de água e de nutrientes, principalmente o $\mathrm{P}$ (Moreira \& Siqueira, 2002).

Quanto ao teor de potássio (K), os melhores resultados $(\mathrm{p}<0,05)$ foram encontrados para os tratamentos $G$. clarum, A. morrowiae e G. clarum + A. morrowiae, com aumento nos teores de K variando de 14,3 a 23,4\%, com- parado à testemunha sem FMA (Tabela 2). Já o teor de magnésio $(\mathrm{Mg})$ e enxofre $(\mathrm{S})$ foi superior $(\mathrm{p}<0,05)$ para o tratamento com A. morrowiae (Tabela 2). Os teores de K encontrados na parte aérea do pinhão-manso, no presente trabalho, foram maiores aos observados em outros experimentos sem utilização de FMA (Laviola \& Dias, 2008; Silva et al., 2010), porém com adubação e omissão de nutrientes, evidenciando a importância de FMA na absorção de K. Para Mg e S, os teores, em média, foram próximos aos observados por Silva et al. (2010) em experimento com adubação completa em casa de vegetação. Assim, mesmo sem adubação completa, a associação micorrízica, utilizando a espécie $A$. morrowiae, proporcionou aumento nos teores de $\mathrm{Mg}$ e S (Tabela 2).

Para os teores de micronutrientes na parte aérea (Tabela 2) não foram encontradas diferenças significativas para ferro $(\mathrm{Fe})$, zinco $(\mathrm{Zn})$ e cobre $(\mathrm{Cu})$. Para os teores de manganês $(\mathrm{Mn})$, o melhor resultado significativo $(\mathrm{p}<0,05)$ foi observado no tratamento inoculado com $A$. morrowiae, com teor acima de $80 \%$ em relação à testemunha sem FMA (Tabela 2). Já nos teores de boro (B), destaque para os tratamentos com A. morrowiae, S. calospora + G. clarum e G. clarum + A. morrowiae, superiores $(\mathrm{p}<0,05)$ aos demais, com incremento nos teores de B variando entre 9 e 15\% em relação a testemunha sem FMA (Tabela 2), indicando a importância da associação entre fungo e planta para a absorção de Mn e B, principalmente pela baixa mobilidade no solo (Colozzi-Filho \& Balota, 1994).

Os resultados mostraram os efeitos positivos observados em relação ao $\mathrm{N}, \mathrm{P}, \mathrm{K}, \mathrm{Mg}, \mathrm{S}, \mathrm{Mn}$ e B da mesma forma como observado por outros autores com outras culturas (Oliveira \& Oliveira, 2004; Pouyu-Rojas et al., 2006), sendo indicativos de alguma contribuição das micorrizas na absorção desses nutrientes.

Tabela 2. Teores de macro e de micronutrientes na parte aérea de mudas de pinhão-manso não inoculadas e inoculadas com fungos micorrízicos arbusculares (FMAs) ${ }^{1}$

\begin{tabular}{|c|c|c|c|c|c|c|c|c|c|c|c|}
\hline \multirow{2}{*}{ Tratamentos } & $\mathbf{N}$ & $\mathbf{P}$ & $\mathbf{K}$ & $\mathbf{C a}$ & Mg & $\mathbf{S}$ & $\mathbf{F e}$ & Mn & B & Zn & $\mathbf{C u}$ \\
\hline & \multicolumn{10}{|c|}{$\mathrm{g} \mathrm{Kg}^{-1}$} & \\
\hline Sem FMAs & $8,6 \mathrm{~b}$ & $1,45 \mathrm{~d}$ & $25,2 \mathrm{~b}$ & $9,16 \mathrm{a}$ & $5,2 \mathrm{c}$ & $0,78 \mathrm{c}$ & $331 \mathrm{a}$ & $700 \mathrm{c}$ & $43,4 \mathrm{~b}$ & $29,7 \mathrm{a}$ & $3,1 \mathrm{a}$ \\
\hline MIX de FMAs & $8,5 \mathrm{~b}$ & $1,83 \mathrm{c}$ & $26,2 \mathrm{~b}$ & $9,23 \mathrm{a}$ & $7,0 \mathrm{~b}$ & $1,07 \mathrm{~b}$ & $335 \mathrm{a}$ & $745 \mathrm{c}$ & $45,5 \mathrm{~b}$ & $23,5 \mathrm{a}$ & $5,3 \mathrm{a}$ \\
\hline Scutellospora calospora & $10,1 \mathrm{a}$ & $1,30 \mathrm{c}$ & $26,2 \mathrm{~b}$ & $8,58 \mathrm{a}$ & $5,6 \mathrm{c}$ & $1,02 \mathrm{~b}$ & $301 \mathrm{a}$ & $929 \mathrm{~b}$ & $41,5 \mathrm{~b}$ & $26,8 \mathrm{a}$ & $4,2 \mathrm{a}$ \\
\hline Glomus clarum & $10,8 \mathrm{a}$ & $2,16 \mathrm{a}$ & $28,8 \mathrm{a}$ & $9,48 \mathrm{a}$ & $6,6 b$ & $0,94 \mathrm{c}$ & $248 \mathrm{a}$ & $735 c$ & $43,8 \mathrm{~b}$ & $22,5 \mathrm{a}$ & $3,2 \mathrm{a}$ \\
\hline Glomus margarita & $7,6 \mathrm{~b}$ & $1,13 \mathrm{~d}$ & $25,5 \mathrm{~b}$ & $8,41 \mathrm{a}$ & $5,2 \mathrm{c}$ & $0,77 \mathrm{c}$ & $328 \mathrm{a}$ & $952 \mathrm{~b}$ & $43,8 \mathrm{~b}$ & $20,8 \mathrm{a}$ & $3,9 \mathrm{a}$ \\
\hline Acaulospora morrowiae & $10,3 \mathrm{a}$ & $2,21 \mathrm{a}$ & $31,1 \mathrm{a}$ & $11,27 \mathrm{a}$ & $8,3 \mathrm{a}$ & $1,60 \mathrm{a}$ & $340 \mathrm{a}$ & $1275 \mathrm{a}$ & $49,6 \mathrm{a}$ & $26,9 \mathrm{a}$ & $4,1 \mathrm{a}$ \\
\hline S. calospora + G. clarum & $7,6 \mathrm{~b}$ & $1,75 \mathrm{c}$ & $26,3 \mathrm{~b}$ & $8,21 \mathrm{a}$ & $5,0 \mathrm{c}$ & $0,69 \mathrm{c}$ & $388 \mathrm{a}$ & $975 \mathrm{~b}$ & $47,3 \mathrm{a}$ & $29,4 \mathrm{a}$ & $4,1 \mathrm{a}$ \\
\hline S. calospora + G. margarita & $8,4 \mathrm{~b}$ & $1,84 \mathrm{c}$ & $23,1 \mathrm{c}$ & $8,33 \mathrm{a}$ & $5,8 \mathrm{c}$ & $0,88 \mathrm{c}$ & $320 \mathrm{a}$ & $811 \mathrm{c}$ & $43,6 \mathrm{~b}$ & $26,4 \mathrm{a}$ & $3,8 \mathrm{a}$ \\
\hline S. calospora + A. morrowiae & $8,5 \mathrm{~b}$ & $2,01 \mathrm{~b}$ & $27,3 \mathrm{~b}$ & $8,57 \mathrm{a}$ & $5,7 \mathrm{c}$ & $0,92 \mathrm{c}$ & $231 \mathrm{a}$ & $787 \mathrm{c}$ & $45,6 \mathrm{~b}$ & $22,8 \mathrm{a}$ & $5,1 \mathrm{a}$ \\
\hline G. clarum + G. margarita & $9,3 \mathrm{~b}$ & $2,23 \mathrm{a}$ & $24,1 \mathrm{c}$ & $8,35 \mathrm{a}$ & $5,3 \mathrm{c}$ & $0,97 \mathrm{~b}$ & $280 \mathrm{a}$ & $798 \mathrm{c}$ & $42,6 \mathrm{~b}$ & $24,0 \mathrm{a}$ & $4,2 \mathrm{a}$ \\
\hline G. clarum + A. morrowiae & $9,1 \mathrm{~b}$ & $2,37 \mathrm{a}$ & $29,4 \mathrm{a}$ & $8,60 \mathrm{a}$ & $5,5 \mathrm{c}$ & $0,87 \mathrm{c}$ & $351 \mathrm{a}$ & $966 \mathrm{~b}$ & $49,8 \mathrm{a}$ & 33,4 a & $3,2 \mathrm{a}$ \\
\hline G. margarita + A. morrowiae & $12,5 \mathrm{a}$ & $1,47 \mathrm{c}$ & $21,8 \mathrm{c}$ & $8,68 \mathrm{a}$ & $5,7 \mathrm{c}$ & $0,95 \mathrm{~b}$ & $336 a$ & $765 c$ & $41,7 \mathrm{~b}$ & $21,1 \mathrm{a}$ & $3,4 \mathrm{a}$ \\
\hline $\mathrm{CV}(\%)$ & 12,6 & 17,2 & 20,7 & 37,2 & 14,9 & 22,9 & 69,7 & 24,1 & 11,9 & 31,8 & 50,8 \\
\hline
\end{tabular}

${ }^{1}$ Médias seguidas por diferentes letras na coluna diferem estatisticamente entre si pelo teste de Scott-Knott a $5 \%$ de probabilidade. CV, Coeficiente de variação. 
Para a massa de matéria seca da parte aérea (MSPA), destaque para os tratamentos com G. clarum, G. margarita, A. morrowiae, S. calospora + G. clarum, $S$. calospora $+G$. margarita, G. clarum + A. morrowiae e G. margarita + A. morrowiae, assim como para o tratamento com MIX de micorrizas, com as maiores médias ( $\mathrm{p}<0,05)$ (Tabela 3) com aumento da MSPA variando de 13 a 23\% em relação à testemunha sem FMA. Incrementos significativos para a biomassa da parte área também foram observados por Balota et al. (2010) para o pinhão-manso inoculado com FMA.

Para a massa de matéria seca da raiz (MSR), as maiores médias $(\mathrm{p}>0,005)$ foram observadas para os tratamentos com G. clarum, A. morrowiae, S. calospora + G. clarum, S. calospora + A. morrowiae e G. clarum + A. morrowiae, com incremento variando de 35 a $41 \%$ em relação à testemunha sem FMA (Tabela 3). Já para a massa de matéria seca total (MST), as maiores médias ( $\mathrm{p}>0,005)$ foram para os tratamentos com G. clarum, A. morrowiae, S. calospora + G. clarum, S. calospora + G. margarita, G. clarum + A. morrowiae e G. margarita + A. morrowiae (Tabela 3), com incremento variando de 23 a $28 \%$ em relação ao tratamento testemunha sem FMA.

A média de colonização das raízes de pinhão-manso inoculado com FMA foi de $54,4 \%$, sendo os valores significativamente superiores $(\mathrm{p}<0,05)$ para os tratamentos com G. clarum, A. morrowiae, S. calospora + G. clarum, S. calospora + G. margarita e G. clarum + A. morrowiae (Tabela 3). Pelos resultados obtidos para todos os tratamentos com FMA, podem ser considerados de médio a alto os níveis de colonização micorrízica acima de 50\%, porém em todos os tratamentos forma encontradas FMA nas plantas de pinhão-manso, amostradas e identificadas através das estruturas (hifas), características provenientes dessa associação. Altas taxas de colonização micor- rízica encontradas nas mudas com 75 dias após a emergência demonstram o importante papel desses fungos para a planta desde a sua formação, confirmando a existência dessa simbiose entre os FMAs e esta espécie de planta, como relatado, também, por Carvalho (2008) e Folli-Pereira et al. (2010).

Para a eficiência micorrízica (EM), que expressa o quanto as plantas micorrizadas foram superiores às não micorrizadas, baseada na massa de matéria seca das plantas como a MSPA, MSR e MST, a micorrização estimulou o desenvolvimento da parte aérea em mais de $20 \%$, com destaque para MSPA e MST nos tratamentos com $G$. clarum, A. morrowiae, S. calospora $+G$. clarum e $G$. clarum + A. morrowiae (Figura 1). Resultados semelhantes foram observados por Balota et al. (2010) em pinhãomanso com doses de fósforo, com incrementos acima de $100 \%$ em doses variando de 0 a $100 \mathrm{mg} \mathrm{kg}^{-1}$.

A ação benéfica dos FMAs sobre a produção de culturas anuais gera incrementos que variam de 5 a $290 \%$, enquanto os benefícios para o crescimento ou produção de mudas variam de $50 \%$ a mais de $1.000 \%$, conforme Siqueira et al. (2002). Dessa forma, as respostas da inoculação de FMA são maiores, mais consistentes e promissoras em plantas que passam por fase de formação de mudas (Siqueira et al., 2002), como ocorre com o pinhãomanso.

No entanto, ainda é limitada a quantidade de informações relacionadas aos níveis de benefícios que essa associação pode trazer para essa espécie, principalmente nas condições da região de cerrado. Além disso, não há conhecimento da diversidade dos FMAs que se associam ao pinhão-manso e sua relação com as condições edafoclimáticas presentes em regiões como o cerrado no Sul do Tocantins, onde esta planta está sendo cultivada.

Tabela 3. Matéria seca da parte aérea (MSPA), da raiz (MSR), total (MST) e percentagem de colonização (CM) de mudas de pinhãomanso não inoculadas e inoculadas com fungos micorrízicos arbusculares (FMAs) ${ }^{1}$

\begin{tabular}{|c|c|c|c|c|}
\hline Tratamentos & $\operatorname{MSPA}(\mathrm{g})$ & $\operatorname{MSR}(\mathbf{g})$ & $\operatorname{MST}(\mathrm{g})$ & CM\% \\
\hline Sem FMAs & $211,5 \mathrm{c}$ & $65,7 \mathrm{~d}$ & $277,2 \mathrm{~d}$ & $0 \mathrm{f}$ \\
\hline MIX de FMAs & $263,5 \mathrm{a}$ & $71,1 \mathrm{c}$ & $334,6 \mathrm{~b}$ & $53,3 \mathrm{~b}$ \\
\hline Scutellospora calospora & $226,0 \mathrm{~b}$ & $76,5 \mathrm{c}$ & $302,5 \mathrm{c}$ & $30 \mathrm{~d}$ \\
\hline Glomus clarum & 259,7 a & $91,8 \mathrm{a}$ & $350,3 \mathrm{a}$ & $64,7 \mathrm{a}$ \\
\hline Glomus margarita & $240,0 \mathrm{a}$ & $87,4 \mathrm{~b}$ & $327,4 \mathrm{~b}$ & $45,7 \mathrm{c}$ \\
\hline Acaulospora morrowiae & $251,2 \mathrm{a}$ & $92,8 \mathrm{a}$ & $344,0 \mathrm{a}$ & $66,7 \mathrm{a}$ \\
\hline S. calospora + G. clarum & 266,7 a & 89,4 a & 356,1 a & $76,7 \mathrm{a}$ \\
\hline S. calospora + G. margarita & $264,5 \mathrm{a}$ & $83,1 \mathrm{~b}$ & 347,6 a & $68,3 \mathrm{a}$ \\
\hline S. calospora + A. morrowiae & $203,7 \mathrm{c}$ & 89,3 a & $293,0 \mathrm{c}$ & $20 \mathrm{~d}$ \\
\hline G. clarum + G. margarita & $225,7 b$ & $84,3 \mathrm{~b}$ & $310,0 \mathrm{~b}$ & $53,7 \mathrm{~b}$ \\
\hline G. clarum + A. morrowiae & 253,7 a & $91,9 \mathrm{a}$ & 345,6 a & $66,3 \mathrm{a}$ \\
\hline G. margarita + A. morrowiae & 256,0 a & $85,3 \mathrm{~b}$ & $341,3 \mathrm{a}$ & $53 \mathrm{~b}$ \\
\hline $\mathrm{CV}(\%)$ & 14,7 & 12,9 & 13,0 & 27,9 \\
\hline
\end{tabular}

${ }^{1}$ Média seguidas por diferentes letras na coluna não diferem estatisticamente entre si pelo teste de Scott-Knott a $5 \%$ de probabilidade. CV, Coeficiente de variação. 


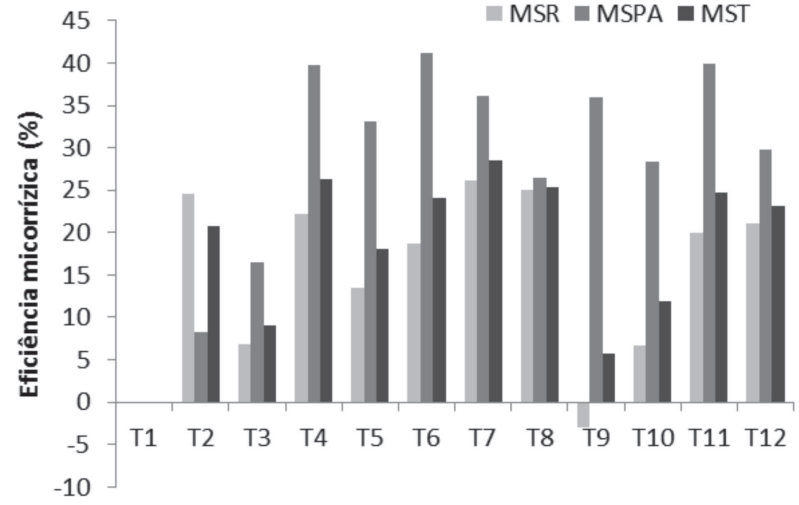

Figura 1. Eficiência dos fungos micorrízicos arbusculares (FMAs) no desenvolvimento da parte aérea e das raízes de pinhão-manso. T1: Testemunha (sem micorriza), T2: Mistura das micorrizas (MIX), T3: Scutellospora calospora, T4: Glomus clarum, T5: G. margarita, T6: Acaulospora morrowiae, T7: S. calospora + G. clarum, T8: S. calospora + G. margarita, T9: $S$. calospora + A. morrowiae, T10: G.clarum + G. margarita, T11: G.clarum + A. morrowiae e T12: G. margarita + A. morrowiae MSR: matéria seca da raiz, MSPA: matéria seca da parte aérea e MST: matéria seca total.

\section{CONCLUSÃO}

A micorrização aumentou a biomassa e os teores de nutrientes das mudas de pinhão-manso, principalmente com a utilização de Glomus clarum e Acaulospora morrowiae, isoladamente e em dupla inoculação. Estas espécies de fungos micorrízicos arbusculares apresentaram eficiência micorrízica para a biomassa das plantas.

\section{AGRADECIMENTOS}

A UFT Campus de Gurupi, CNPq e CAPES pelos recursos referente ao Processo no 473389/2007-0 e CAPES PNPD.

\section{REFERÊNCIAS}

Balota EL, Machineski O, Truber PV, Cerezini P, Milani KL, Scherer A, Honda C \& Leite LG (2010) Desenvolvimento e nutrição de pinhão-manso inoculado com fungos micorrízicos arbusculares em diferentes doses. In: IV Congresso Brasileiro de Mamona e I Simpósio Internacional de Oleaginosas Energéticas, João Pessoa. Anais, p.605-609.

Bremner JM \& Mulvaney CS (1982) Total nitrogen. In: Page AL (Ed.) Methods of soil analysis. Madison, American Society of Agronomy. p.595-694.

Carvalho AMX (2008) Fungos micorrízicos arbusculares no crescimento de mudas de pinhão-manso (Jatropha curcas L.). Dissertação de Mestrado. Universidade Federal de Viçosa, Viçosa, 76p.

Colozzi-Filho A \& Balota EL (1994) Micorrizas arbusculares. In Hungria M \& Araújo RS (Eds.) Manual de métodos empregados em estudos em microbiologia agrícola. Brasília, EMBRAPA. p.383-418.

Dias LAS, Leme LP, Laviola BG, Pallini Filho A, Pereira OL, Carvalho M, Manfio CE, Santos AS, Sousa LCA, Oliveira TS \& Dias DCFS (2007) Cultivo do pinhão-manso (Jatropha curcas L.) para produção de óleo combustível. Viçosa, UFV. 40p.
Folli-Pereira MSF, Rodrigues AL, Haddad LSM, Moreira BC, Serafini M, Bazzolli DMS \& Kasuya MCM (2010) Micorriza arbuscular em plantas de pinhão-manso durante o estresse hídrico. In: FertBio, Guarapari. Anais, FertBio. CD-ROM.

Francis G, Edinger R \& Becker K (2005) A concept for simultaneous wasteland reclamation, fuel production, and socio-economic development in degraded areas in India: Need, potential and perspectives of Jatropha plantations. Natural Resources Forum, 29:12-24

Giovanetti M \& Mosse B (1980) An evaluation of techniques for measuring vesicular arbuscular mycorrhizal infection in roots. New Phytologist, 84:489-500.

Kiriachek SG, Azevedo LCB, Peres LEP \& Lambais MR (2009) Regulação do desenvolvimento de micorrizas arbusculares. Revista Brasileira de Ciência do Solo, 33:1-16.

Lamothe PJ, Fries TL \& Consul JJ (1986) Evaluation of microwave oven system for the dissolution of geologic samples. Analytical Chemistry, 58:1881-1886.

Laviola BG \& Dias LAS (2008) Teor e acúmulo de nutrientes em folhas e frutos de pinhão-manso. Revista Brasileira de Ciência do Solo, 32:1969-1975.

Miranda JCC (2008) Cerrado: Micorriza arbuscular - ocorrência e manejo. Planaltina, Embrapa Cerrados. 169p.

Moreira FMS \& Siqueira JO (2002) Microbiologia e bioquímica do solo. Lavras, Universidade Federal de Lavras. 625p.

Oliveira AN \& Oliveira LA (2004) Associação micorrízica e teores de nutrientes nas folhas de cupuaçuzeiro (Theobroma grandiflorum) e guaranazeiro (Paullinia cupana) de um sistema agroflorestal em Manaus, Amazonas. Revista Brasileira de Ciência do Solo, 28:1063-1068.

Pouyu-Rojas E, Siqueira JO \& Santos JGD (2006) Compatibilidade simbiótica de fungos micorrízicos arbusculares com espécies arbóreas tropicais. Revista Brasileira de Ciência do Solo, 30:413424.

Silva EB, Tanure LPP, Souza PT, Grazziotti PH \& Silva AC (2010) Crescimento de pinhão-manso em neossolo quartzarênico usando a técnica do nutriente falante. Revista Brasileira de Oleaginosas e Fibrosas, 14:73-81.

Siqueira JO, Lambais MR \& Stürmer SL (2002) Fungos micorrízicos arbusculares. Biotecnologia Ciência \& Desenvolvimento, 25:1221

Rev. Ceres, Viçosa, v. 59, n.1, p. 142-146, jan/fev, 2012 